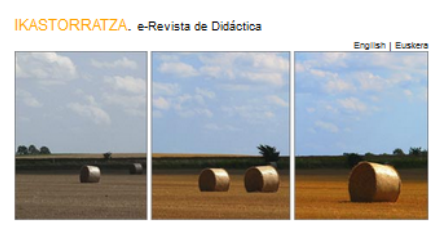

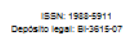

Inicio, Sobre nosotros, Publicacionesı, Participa

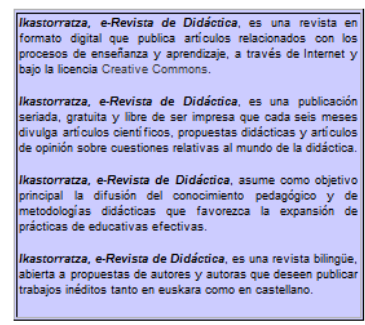

\section{IKASTORRATZA. Didaktikarako e-aldizkaria}

\author{
IKASTORRATZA. e-journal on Didactics
}

ISSN: 1988-5911 (Online) Journal homepage: http://www.ehu.eus/ikastorratza/

La flexión nominal en la producción escrita de estudiantes benineses del profesorado de ELE

\author{
IRASTORRATZA. e-Revista de \\ Didáctica
}




\title{
La flexión nominal en la producción escrita de estudiantes benineses del profesorado de ELE
}

\author{
Laurent-Fidèle Sossouvi \\ Université d’Abomey-Calavi (Benín) \\ laurent.sossouvi108@gmail.com \\ Mei-Chih Lin \\ Université d’Abomey-Calavi (Benín) \\ linmeichih@hotmail.com
}

\section{Resumen}

Este trabajo tiene como objetivo examinar el nivel de dominio de conocimiento de las construcciones de la concordancia nominal en estudiantes benineses para profesores de ELE y comprobar si existen diferencias en función del sexo y el estatuto del becario de Estado o no. Para dar respuesta a estos objetivos se utilizaron un cuestionario y actividades escritas para recoger las opiniones y producciones escritas de 45 participantes universitarios multilingües que están en cuarto semestre del Grado. Los resultados obtenidos, tras un análisis mixto, muestran los tipos y causas importantes de los errores, encontrando diferencias por sexo y estatuto. Finalmente, se realizan sugerencias para mejorar el diseño de la formación inicial del profesorado de ELE y contribuir a la mejora de la educación.

Palabras clave: contacto de lenguas; discordancia gramatical; detección de errores; adquisición de ELE; atención a la forma; influencia translingüística. 


\begin{abstract}
This paper aims to examine the level of knowledge mastery of nominal concordance constructions in Beninese students in teaching Spanish as a foreign language and check if there are differences according to sex and status of government grant holder or not. To meet these objectives we used a questionnaire and written activities with a sample of 45 multilingual students in their fourth semester of studies, to collect their opinion and written production. The results obtained, after a mixed analysis, show the important types and causes of errors, finding differences by sex and student status. Finally, suggestions are made to improve the design of Spanish secondary school teachers' initial training and contribute to instructional improvement.
\end{abstract}

Keywords: language contact; grammatical discordance; error detection; acquisition of Spanish as a Foreign Language; focus on form; translinguistic influence. 


\section{Introducción}

Han sido varios los estudios (Sossouvi, 2004, 2014, 2016ab; Djandué, 2012; Yao, 2015, entre otros) sobre la adquisición del español como lengua no materna en el África francófona, sobre todo en Benín; sin embargo, hoy en día, se conoce muy poco acerca del uso de la flexión nominal por los estudiantes universitarios. Se admite comúnmente que el dominio de esta propiedad gramatical es asequible; sobre todo para aprendientes cuya primera lengua (L1) es estructuralmente afín a la lengua meta, como en nuestro caso. También dentro de los contenidos gramaticales enseñados en las clases de español como lengua extranjera (ELE,) la flexión nominal parece ser uno de los que poseen menos complejidad. Ahora bien, las marcas de género y número varían de una lengua a otra, en el tiempo e incluso de una región a otra. Además, no es raro que tanto hablantes nativos como no nativos cometan errores de concordancia (por ejemplo, Dewaele y Véronique, 2001; Ayoun, 2010; Lew-Williams y Fernald, 2010). En la misma línea, muchos estudiosos (Montrul, de la Fuente, Davidson y Foote, 2013; Ferreira Cabrera y Elejalde Gómez, 2017; Gonzalez, Mayans y van den Bergh, 2019), han señalado que estas dos categorías morfosintácticas engendran escollos sempiternos para los discentes de segunda lengua (L2) y lengua extranjera (LE).

Nuestra propuesta responde a la necesidad de llenar ese vacío y examina el nivel de dominio de conocimiento de las construcciones de la concordancia nominal en estudiantes benineses para profesores de ELE, con el fin de plantear sus dificultades en el uso de estas marcas gramaticales, intentar explicar las fuentes de las incorrecciones, así como identificar algunas implicaciones didácticas y curriculares. Nuestra hipótesis al respecto sería que el usuario de la LE, aunque haya llegado a una etapa avanzada en su proceso adquisicional, sigue cometiendo errores en los usos de la flexión nominal. Igualmente, presumimos que los participantes becarios de Estado aciertan más que los 
estudiantes no becarios, y conociendo el contexto de aprendizaje-enseñanza, que las mujeres fallan más que los hombres. Para conocer la jerarquía de sus dificultades nos apoyamos en los paradigmas del análisis contrastivo y del análisis de errores.

La flexión nominal expresa género y número mediante marcas femeninas y plurales. Comprende los sustantivos, adjetivos cualitativos, ciertos determinantes y pronombres. No obstante, cabe mencionar que en este trabajo solo nos centraremos en los primeros. Los becarios de Estado son bachilleres que disfrutan de una beca para estudios, tras aprobar el concurso de ingreso a la escuela de formación del profesorado de educación secundaria. Las becas se otorgan en base a necesidad y mérito, a ciudadanos benineses que tienen como máximo 21 años el 31 de diciembre del curso; titulados con promedio mínimo de 12/20.

El artículo está organizado de acuerdo con el siguiente esquema: la primera sección presenta algunas consideraciones sobre la flexión nominal de tres lenguas del repertorio lingüístico de los participantes de la investigación. A continuación, revisamos las publicaciones disponibles sobre las dificultades en esta categoría gramatical por parte de aprendientes de otras lenguas. En la segunda sección se expone el marco metodológico del trabajo; describiendo el perfil de los informantes, los materiales y procedimientos utilizados. La tercera sección presenta los hallazgos significativos y la sección final discute los resultados obtenidos, resume las propuestas realizadas y sugiere algunas consecuencias para la enseñanza de la lengua española. 


\section{Algunas consideraciones previas}

\subsection{Generalidades sobre el género y el número en español, francés y fon}

Benín es un país multilingüe de África Occidental que logró su independencia de Francia en 1960. El francés es la única lengua oficial y la lengua de comunicación interétnica. Asimismo, es la lengua de instrucción desde la educación infantil hasta los estudios de doctorado. En este país africano, se hablan aproximadamente 73 lenguas locales (Sossouvi, 2014: 101). El fon o fongbe es la lengua nacional no oficial más hablada del país. Es la lengua local vehicular hablada por más de la mitad de la población beninesa. Pertenece al grupo kwa de la rama nigero-congoleña, según la clasificación de Greenberg (1963), y se inscribe en el subgrupo gbè (lengua o enunciado).

El español y el francés son lenguas romances, flexionales como la lengua portuguesa. Estas lenguas expresan la variación de palabras relacionadas con categorías como tiempo, persona, género, etc., por desinencias o marcas integradas en palabras. El fongbe tiene poca morfología, resultando en ese aspecto más simple que el español o el francés. Sus nominales y sus verbales no tienen ningún cambio morfológico; tampoco tienen flexiones de modo o de tiempo, ni modificaciones de género y número, ya que todos estos aspectos se indican más bien por marcas o partículas antepuestas o pospuestas (Fadaïro, 2001: 20). Por razones obvias, no se trata de hacer estudios detallados, ni examinar todos los casos (por ejemplo, las descripciones de la flexión de número en compuestos, entre otros); solo daremos una visión general de los usos.

\section{Sobre el género en sustantivos}


En español, la mayoría de los sustantivos acabados en -o son masculinos y la mayoría de los terminados en -a son femeninos: el plato, el metro; la casa, la cena. Existen, no obstante, varias excepciones:

-Hay sustantivos masculinos acabados en -a (el problema, el mapa); y sustantivos femeninos en -o (la mano, la foto).

-Existen sustantivos referidos a persona que tienen la misma forma en masculino que en femenino: los acabados en -ista (el/la deportista) y algunos terminados en -e (el/la cantante). No obstante, se dice: el jefe / la jefa; el sastre / la sastra.

En cuanto a la formación del femenino en francés, la regla general para los sustantivos animados, basta añadir una $-e$ a la forma escrita del masculino; de igual manera que en castellano se agrega -a al masculino: un Espagnol (un español), une Espagnole (una española); un ami (un amigo), une amie (una amiga). Con todo, si el sustantivo ya termina en -e, la forma femenina es igual al masculino y solo el determinante indica el género: un élève (un alumno), une élève (una alumna). El género de los sustantivos inanimados resulta arbitrario y viene dado por el determinante que lo precede. También las terminaciones pueden indicar el género de los sustantivos, a pesar de que hay muchas excepciones. Los sustantivos que terminan en -isme (le patriotisme [el patriotismo]); ment (le parlement [el parlamento]); -(e)au, (le bureau [el despacho], le noyau [el núcleo, el hueso]), etc., son de género masculino.

Son femeninos los sustantivos que terminan en -té (la quantité [la cantidad]); -ion (la solution [la solución]); -ie (la sociologie [la sociología]); etc.

Por otra parte, cabe subrayar que existen diferencias de género entre el español y el francés. Algunos ejemplos: el calor (la chaleur), el dolor (la douleur), la nube (le nuage), 
el periodo (la période), etc. También los nombres de ríos, mares y montañas son masculinos en castellano. Por ejemplo: el Sena (la Seine); los Pirineos centrales (Les Pyrénées centrales).

En lo que atañe al fongbe, generalmente, pocas palabras tienen un masculino diferente del femenino. Muchos sustantivos funcionan como epicenos; es decir, un gran número de sustantivos designa de igual manera a seres animados (personas y animales) pertenecientes a ambos sexos. A título de ejemplo, la palabra v̌̌ es un sustantivo epiceno que se utiliza para designar tanto al niño, al hijo como a la niña, la hija; nəví (el hermano, la hermana); kokló (el gallo, la gallina).

Sin embargo, cuando se quiere distinguir entre los sexos, y cuando las circunstancias lo requieran, se puede agregar la palabra súnnù (hombre), nyว̆nù (mujer) para los seres humanos. Por poner algunos ejemplos: v̌̌ súnnù (el niño varón, el niño), v̌̌ nyว̆nù (la niña); nəví súnnù (el hermano varón, el hermano), nəví nyว̌nù (la hermana).

De igual manera para diferenciar el sexo de los animales, se utilizan los términos asú (el macho, el masculino, el esposo) o asì (la hembra, el femenino, la esposa) siguiendo al sustantivo epiceno. Así, por ejemplo, diríamos kokló asú (el gallo), kokló asì (la gallina);

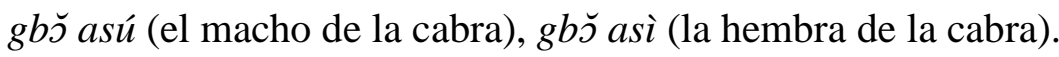

$\mathrm{Si}$, por otra parte, el sustantivo designa una planta con flores masculinas o flores femeninas, la diferencia de sexo se marca mediante la oposición asú (macho), asì (hembra): kpèntín (la papaya); kpèntín asú (la papaya con flores masculinas), kpèntín asì (la papaya con flores femeninas).

\section{Sobre el número en sustantivos}


Las tres lenguas tienen su modo de formar el plural de los sustantivos. En español, la mayoría de los sustantivos forman el plural con -s o -es, según los casos; y los terminados en vocal añaden -s. Ejemplos: casa / casas; libro / libros. Pero: esquíes o esquís, tabúes o tabús. A los sustantivos singulares que terminan en consonante o -y, se les agrega -es para formar el plural: el papel/los papeles; el rey/ los reyes. Los nombres terminados en dos o más consonantes forman el plural en -s (salvo los acabados ya en -s): estand(s). Los que acaban en -s y en sílaba no acentuada, tienen igual forma en singular y plural.

Por regla general el plural se forma en francés añadiendo una -s al singular del sustantivo: un enfant [un niño], des enfants [niños, hijos]; une banane [un plátano], des bananes [plátanos]. No obstante, existen muchos casos especiales. Los sustantivos que en singular terminan en $-s,-\chi$ o $o-z$ no modifican. Ejemplos: le pays [el país], les pays [los países]; le vieux [el viejo], les vieux [los viejos]; le gaz [el gas], les gaz [los gases].

Los sustantivos que acaban en -eau, -au o -eu, se forman el plural al añadir una "x": le gâteau [el pastel, la tarta], les gâteaux [los pasteles]; le tuyau [la manguera], les tuyaux [las mangueras]; le feu [el fuego], les feux [los fuegos]. Existen algunas excepciones: le pneu [el neumático], les pneus [los neumáticos]; le bleu [el azul], les bleus [los azules].

Hay siete sustantivos que terminan en -ou y que toman - $x$ en plural: bijou(x) [joya(s)], caillou(x) [piedra(s), guijarro(s), pedrusco(s)], chou(x) [repollo(s), col(es)], genou(x) [rodilla(s)], hibou(x) [búho(s)], joujou(x) [juguete(s)], pou(x) [piojo(s)].

También una decena de sustantivos acabados en -ail cambian esta terminación por -aux en plural: travail, travaux [trabajo(s)]; corail, coraux [coral(es)], etc. Las otras palabras en -ail siguen la regla general añadiendo una $s$. 
Una treintena de sustantivos terminados en -al, forman el plural con -aux. Algunos ejemplos: local, locaux [local(es)]; métal, métaux [metal(es)], etc.

Las otras palabras siguen la norma general añadiéndose una -s: le bal, les bals [los bailes]; le carnaval, les carnavals [los carnavales], etc.

La lengua francesa tiene algunos plurales irregulares: œil [ojo], yeux [ojos]; jeune homme [joven], jeunes gens [los jóvenes, la gente joven]; ciel [cielo], cieux [cielos], etc.

En cuanto al fongbe, el plural de los sustantivos no existe (Fadaïro, 2001: 25; Société des Missions Africaines, 2012: 23). Aun así, la idea de pluralidad se forma agregando al sustantivo, que permanece invariable, la partícula lé. Por ejemplo: yəkpóvú (el niño, la niña), yっkpóvú lé (los niños, las niñas); nyŏnù (la mujer), nyŏnù lé (las mujeres).

Cuando el sustantivo va acompañado de un adjetivo, la marca del plural lé se coloca después de este adjetivo que sigue inmediatamente al sustantivo. Citamos como ejemplo: atín daxó (el gran árbol), atín daxó lé (los grandes árboles); só kpeví (el pequeño caballo), só kpeví lé (los pequeños caballos).

Cuando hay una palabra que expresa una idea de pluralidad, como un adjetivo numeral o un adverbio de cantidad, este adjetivo o adverbio se coloca después del sustantivo que determina o modifica y la marca lé se omite o se suprime. A título de ejemplo, kokló

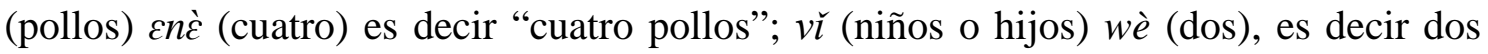
niños o dos hijos.

Por el contrario, cuando el adjetivo numeral expresa la totalidad, lé se coloca después de este adjetivo. Por ejemplo, kokló (pollos) cnè (cuatro) lé (los), es decir “los cuatro pollos”;

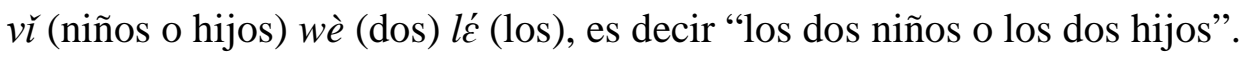


En síntesis, el francés y el español tienen dos posibilidades para expresar el género gramatical, es decir el masculino y el femenino; dos posibilidades para expresar el número gramatical, así como concordancias gramaticales de número y género, dependiendo normalmente de las terminaciones o de las categorías de los sustantivos. El plural de los sustantivos en ambas lenguas se forma, por regla general, añadiendo una -s al singular y existen algunos plurales irregulares. Aun así, en español, el género y el número de sustantivos se forma de modo diferente que en francés. Estas dos lenguas fusionantes, tienen en común muchas características gramaticales. Por consiguiente, se espera que se van a producir transferencia positiva e interferencia entre ellas.

En comparación con las dos lenguas romances, el fongbe no tiene cambios ni de género ni de número. El sustantivo no varía, es decir no conoce flexiones y guarda la misma forma en masculino o en femenino, en singular o en plural. En cambio, existen distintos procedimientos sencillos para expresar todos estos matices. 


\subsection{Estado de la cuestión}

La investigación sobre el proceso de adquisición de la flexión nominal en español como L2 o LE ha estado dominada considerablemente por el estudio de la concordancia de género y número en la interlengua de los aprendientes de LE, así como las propiedades que se derivan del género gramatical (Rothman, González Alonso y Miller, 2018).

Torijano (2008) investiga la adquisición de los determinantes españoles en la producción escrita de estudiantes lusohablantes. Su propósito es conocer las zonas de mayor dificultad entre los determinantes, basándose en los paradigmas del análisis contrastivo, el análisis de errores y la interlengua. Los resultados revelan que la proximidad lingüística de ambas lenguas dificulta el conocimiento real de la lengua meta.

En la misma línea, Ferreira Cabrera y Elejalde Gómez (2017) analizan el CAELE, una colección de 418 textos escritos producidos por 62 estudiantes de ELE. Su meta es determinar los errores más frecuentes y recurrentes de ese corpus. Los resultados muestran que los fallos más usuales y reiterados en este corpus son los de falsa selección de género gramatical y la omisión de la tilde ortográfica. Igualmente, esos hallazgos sugieren el tratamiento de los errores según el nivel de competencia en el proceso de corrección por escrito.

Por otra parte, Zuheros Garrido y Marrero Aguiar (2019) estudian la adquisición de la concordancia nominal de género y número en tres grupos de estudiantes sinohablantes (90 niños, 90 adolescentes y 90 adultos), todos de nivel inicial, además de centrarse en el comportamiento de la variable edad. Los estudiantes realizan tres pruebas diferentes de descripción de láminas, de complejidad creciente, en tres periodos lectivos trimestrales. Los resultados indican que los tres grupos de edad presentan una mayor tasa de aciertos que de errores en esta categoría gramatical y la edad de los aprendientes condiciona el 
proceso de adquisición de la concordancia de género, ya que los niños presentan una mayor tasa de errores que los adolescentes, y estos más que los adultos. Mientras que la concordancia de número no presenta este patrón, tiene una tasa de error semejante en los tres grupos: ambos autores achacan este último hallazgo a las horas de instrucción, similares para todos ellos. En consecuencia, su hipótesis de partida fue confirmada parcialmente: en la concordancia de género se confirma, pero en la de número no.

En la literatura consultada, hemos observado que tanto hablantes nativos de español como hablantes no nativos cometen errores en la flexión nominal en español y también cuando los primeros viven en una situación bilingüe y bicultural o cuando están aprendiendo otra LE también meten la pata en estas categorías gramaticales.

Así lo indican los datos recogidos por Eddington y Hualde (2008) que señalan los errores de hablantes nativos de español a la hora de asignar género a ciertos sustantivos femeninos en español. La variación en el uso de modificadores prenominales masculinos produce confusión en estos nativos: “Echa todo el agua fría en el barreño” (Eddington y Hualde, 2008: 4) (cf. Echa toda el agua fría).

Otros autores tales como Smead (2000), Aaron (2015) han demostrado que los hablantes bilingües de español y otra lengua tienen una preferencia similar para asignar el género masculino a los determinantes de préstamos (Smead, 2000; Aaron, 2015), con la excepción de los préstamos establecidos que están fuertemente integrados morfológicamente en español como “¿Una troca verde?” (Clegg y Waltermire, 2009: 2). En otra investigación Parafita Couto, Munarriz, Epelde, Deuchar y Oyharçabal (2015) examinan las estrategias gramaticales de asignación de género de las frases sustantivas en español-vasco. Para recoger los datos, utilizan un enfoque de tareas múltiples. Los resultados indican una preferencia por el determinante femenino cuando es congruente 
con la terminación fonológica vasca -a, proporcionando evidencia convergente del papel de la forma fonológica de un sustantivo en la asignación de género. Su hallazgo difiere de la tendencia masculina predeterminada observada en los bilingües español-inglés de Jake, Myers-Scotton y Gross (2002).

En un estudio similar, Bellamy, Parafita Couto y Stadthagen-Gonzalez (2018) examinan la asignación de género en frases sustantivas en español-purépecha utilizando una tarea de producción y una tarea de juicio de aceptabilidad en línea. En la tarea de producción, los 11 participantes bilingües purépecha-español prefieren abrumadoramente usar determinantes masculinos, independientemente de la terminación del sustantivo -o. En la tarea de juicio de aceptabilidad, los participantes también prefieren la asignación masculina, excepto en los casos en que los sustantivos terminaban en -a. Sus resultados indican la influencia de la ortografía del purépecha, mostrando que la ortografía puede llevar a los hablantes bilingües a reinterpretar el sufijo -a final, un marcador de género femenino. Además, los resultados discrepantes de estas tareas proporcionan evidencia de que la modalidad de la tarea puede influir en las estrategias de concordancia de género en hispanohablantes.

Del mismo modo, Beatty-Martínez y Dussias (2019) descubren que las estructuras nominales con alternancia español-inglés son abundantes en el habla de los hablantes bilingües, porque cuando estos últimos hablan toman decisiones oportunistas para poner en marcha los dos (o más) sistemas. El objetivo de su trabajo es determinar en qué medida los patrones de cambio de códigos son específicos de la comunidad o generalizables en diferentes comunidades de habla hispana. Por ello ha diseñado un paradigma conversacional para obtener muestras de habla espontánea de hablantes bilingües. Para maximizar la validez ecológica, los participantes son libres de usar la lengua que quiera. 
Los resultados indican una diferencia subyacente en la representación y el procesamiento del género masculino y femenino en español.

Los hispanohablantes que estudian una LE cometen asimismo errores en las mismas categorías gramaticales. Franco (1999) aplica pruebas a 24 aprendientes españoles de 67 años, que ya llevan tres o cuatro años estudiando en un colegio francés. Los resultados revelan que cuanto mayor es el conocimiento de la lengua meta menor es el número de interferencias. Pero a pesar de empezar a estudiar la LE a muy temprana edad, su L1 sigue teniendo más peso, y sus dificultades en la concordancia de género en francés no se achacan solamente a las interferencias, sino también a la ignorancia de las reglas gramaticales, distracciones, etc.

Destaca el ejemplo de Martín, un inglés que emigró a Argentina a los 17 años. Después de 24 años en Argentina fue entrevistado por Franceschina (2001). Su esposa argentina e hijos hablan español, tiene una vida laboral y cultural en su nuevo país de acogida. La autora grabó su producción oral en español durante 94 minutos, luego la transcribió, la codificó y la analizó. Los resultados indican que Martín, a pesar de su nivel muy avanzado en español, su excelente dominio del español y de haber vivido más de dos décadas en un contexto de inmersión lingüística y cultural; seguía cometiendo errores gramaticales de género y número en oraciones nominales, mostrando que este fenómeno de estancamiento, la fosilización, sobreviene en cualquier etapa del aprendizaje de una L2 o LE.

En resumidas cuentas, estos estudios apuntan que la experiencia lingüística tanto monolingüe como bilingüe o multilingüe puede conducir a las mismas consecuencias adaptativas en el proceso de adquisición de la flexión nominal. 


\section{Datos y metodología de trabajo}

\subsection{Perfil de los informantes}

Los informantes han sido 45 estudiantes benineses de la Escuela de Formación del Profesorado de Educación Secundaria de Porto-Novo; 28 mujeres y 17 hombres, de edades comprendidas entre los 18 y 27 años. Proceden de los doce departamentos del país, de diversos orígenes económicos, sociales, geográficos y lingüísticos. Entre las mujeres, seis (06) eran becarias (su edad media era 20,16 años); dos mujeres casadas (de 23 años y 27 años) y entre los hombres cuatro (04) eran becarios del Estado (su edad media era 20,50 años). Como se observa, las mujeres representaban el 62,22\% de los matriculados en estudios hispánicos. Son francófonos nativos y hablan en casa, en familia lenguas nacionales como fongbe, gungbe, yoruba, nago, adjagbe, saxwegbe, gengbe, torigbe, mahigbe, dendi, lokpa, pila pila, ditamari, bariba, tchabe, peulh, wemegbe, etc. Tienen conocimientos de inglés, de castellano y portugués como lenguas extranjeras. Cabe señalar que cada participante habla en casa o en familia cuatro o cinco de las lenguas locales mencionadas. Además, los participantes aprendieron las lenguas locales en el ámbito familiar y en su mayoría, las aprendieron oralmente, con escasa o nula exposición a las formas escritas. Estos futuros profesores son multilingües experimentados y han adquirido previamente todas estas lenguas antes de comenzar el aprendizaje de la lengua cervantina, como verdaderos principiantes. En el curso de esta investigación estaban cursando el segundo curso del Grado (el cuarto semestre), así que poseen un nivel mínimo A2 o B1 de español (en el Marco Común Europeo de Referencia para las lenguas (MCER)). Según los datos recogidos empezaron el aprendizaje del español desde la clase de Quatrième (el tercer año de educación secundaria) para algunos o desde Seconde (el quinto año de educación secundaria) para otros. Dicho brevemente, el proceso de 
enseñanza-aprendizaje de ELE se realiza en un contexto institucional de instrucción formal. El cuerpo docente está integrado por docentes e investigadores seleccionados en base a sus conocimientos profesionales, antigüedad y aptitud por la docencia.

\subsection{Materiales y procedimiento utilizados}

Este estudio surge de nuestras observaciones directas en el aula y fuera de ella como docentes investigadores universitarios. Los materiales de estudio, recogidos en el mes de noviembre de 2018, provienen de un cuestionario y de cinco de las tareas que realizaron los informantes con el fin de obtener datos concretos sobre el tema. La administración de estos solo tardó una hora y media. Las instrucciones fueron proporcionadas verbalmente y por escrito. A cada participante se le brindó una breve explicación del estudio, la definición de la flexión nominal y se les proporcionó las pruebas en horas de clases. También nos aseguramos durante este estudio de que los docentes nunca habían enseñado los elementos gramaticales seleccionados durante el curso académico. Para incrementar la fiabilidad de la medición y determinar los aciertos y errores las tareas fueron tomadas de Aragonés y Palencia (2009) con sus claves de respuestas y, además nos basamos en las posturas académicas en Bosque y Demonte (1999); RAE y ASALE (2010); Gómez Torrego (2011); RAE (2018), entre otros.

El cuestionario consiste en un conjunto de preguntas sobre los aspectos sociodemográficos de los participantes y sobre su percepción respecto al uso de la flexión nominal. La primera actividad consistía en escribir el artículo correspondiente delante de 37 sustantivos. La segunda tarea estribaba en dar la forma femenina de 19 palabras. La tercera radicaba en poner en plural 20 palabras según la regla conveniente. La cuarta residía en rodear la forma correcta de 21 oraciones. La última tarea consistía en sustituir 
las palabras subrayadas por una sola palabra (seis ítems). Los 103 ítems de las cinco tareas representan diferentes grados de complejidad, la de mayor dificultad es la actividad 2 en la cual, si no hay la forma femenina, se usa la palabra "hembra”. Cabe señalar que pese a que los ítems se presentaron de manera descontextualizada se comprendía. Asimismo, hemos tenido en cuenta la accesibilidad del vocabulario utilizado y la cercanía a la lengua francesa. Hemos usado más de un test con el fin de conseguir unos datos más fiables, puesto que, si solo usamos uno, ese test podría no ser suficiente. Luego, revisamos las fichas de respuestas; identificamos, fichamos y clasificamos los errores. Por último, para interpretar correctamente los fallos de los participantes, utilizamos tanto la interpretación admisible (plausible interpretation) como la interpretación autorizada (authoritative interpretation) (Corder, 1981: 37-38). Efectivamente, nos basamos en el contexto con el fin de tener una reconstrucción admisible y asimismo consultamos a la mitad de los participantes para que nos dieran ellos mismos la(s) causa(s) de sus errores para obtener una reconstrucción autorizada. Debido al tipo de muestra con el que se trabajó, los resultados solo pueden generalizarse para los informantes encuestados.

\section{Exposición de algunos hallazgos relevantes de la investigación}

El objetivo de esta sección es el de presentar los resultados relevantes de las actividades que realizamos en clase para averiguar su dominio de la flexión nominal.

\subsection{Percepciones sobre la concordancia de género y número}

La respuesta de los participantes sobre la pregunta: Para ti, la concordancia en español, ¿es fácil o difícil? ¿Por qué? 
Tabla 1. Recapitulación de resultados sobre la percepción respecto del uso de la flexión nominal

\begin{tabular}{|c|c|c|c|c|c|}
\hline & & Fácil & Difícil & Ambos & Total \\
\hline $\begin{array}{l}\text { Mujeres } \\
\text { becarias }\end{array}$ & & 05 & 01 & 00 & 06 \\
\hline $\begin{array}{l}\text { Mujeres } \\
\text { becarias }\end{array}$ & no & 13 & 05 & 03 & 21 \\
\hline $\begin{array}{l}\text { Hombres } \\
\text { becarios }\end{array}$ & & 03 & 00 & 01 & 04 \\
\hline $\begin{array}{l}\text { Hombres } \\
\text { becarios }\end{array}$ & no & 10 & 03 & 01 & 14 \\
\hline Total & & 31 (68,89\%) & 09 (20\%) & $05(11,11 \%)$ & 45 (100\%) \\
\hline
\end{tabular}

Como se observa en la tabla 1, para la mayoría de los participantes (68,89\%), esta área de la gramática resulta asequible. El 20\% de los participantes percibieron dificultades para usar este aspecto gramatical mientras que el 11,11\% tienen una opinión ambivalente. Cabe señalar que algunos informantes tienen la impresión de no tener medios de control, y cierta frustración debida a la ausencia de reglas claras y límpidas.

\section{2. Errores cometidos en la concordancia de género y número}

Se han registrado, en total, 2.342 errores (50,53\%) relacionados con la flexión nominal, con una media de 52,04 por estudiante, lo que supone una cifra relativamente alta con respecto al número de ítems que aparece en nuestro corpus, que se eleva a 4.635. Se han localizado 435 errores en los becarios y 1.907 en los no becarios. La tabla 2 muestra el número de errores y porcentaje por prueba. 
Tabla 2. Número de errores y porcentaje por prueba

\begin{tabular}{lcccccc}
\hline & Prueba 1 & Prueba 2 & Prueba 3 & Prueba 4 & Prueba 5 & Total \\
\hline $\mathrm{N}^{\mathrm{o}}$ de & 602 & 672 & 667 & 322 & 79 & 2342 \\
errores & & & & & & \\
$\%$ & $25,71 \%$ & $28,69 \%$ & $28,48 \%$ & $13,75 \%$ & $3,37 \%$ & $100 \%$ \\
\hline
\end{tabular}

Prueba 1: Escribe el artículo correspondiente delante de estos sustantivos.

Los 602 errores detectados en la primera prueba se reparten de la manera siguiente: los becarios, 102 errores; los no becarios, 500 errores. Los cinco ítems que resultan más dificultosos, clasificados por orden descendente, son:

(1) diabetes (francés le diabète; cf. la diabetes).

(2) postal (cf. la postal)

(3) nao (cf. la nao)

(4) miel (francés le miel ; cf. la miel)

(5) serpiente (francés le serpent; cf. la serpiente)

De los 45 participantes, 41 han elegido el artículo masculino en lugar del femenino en el caso de (1) y uno no ha hecho ninguna elección. Esta elección errónea del determinativo artículo se debe a la imposición del género de la palabra francesa por asociación.

Treinta y siete (37) participantes han errado en el caso de (2), seleccionando el artículo masculino en lugar del femenino. 
Hemos encontrado en el caso de (3) 36 errores, de los cuales 35 se refieren al uso del artículo masculino en lugar del femenino y uno (1) ha sido dejado en blanco. Los fallos (2) y (3) pueden atribuirse a la simplificación de la regla morfológica.

Se han registrado 34 errores en el caso de (4), algunos informantes seleccionaron erróneamente el artículo masculino en vez del femenino.

Treinta y cuatro (34) participantes han fallado al contestar al caso de (5), optando por el artículo masculino en lugar del femenino. Los casos de (4) y (5) resultan de la imposición del género de la palabra francesa por asociación.

Prueba 2: Da la forma femenina de las siguientes palabras. Si no la hay, usa la palabra hembra.

El corpus ofrece 672 errores cometidos en la forma femenina de las palabras, arrojando un porcentaje del 28,69\% del total de errores realizados por los becarios (143 errores) y de los no becarios (529 errores). Los siete ítems más problemáticos se enumeran a continuación en orden descendente.

(6) El carnero (cf. La cordera)

(7) El testigo (cf. La testigo)

(8) El tiburón (cf. El tiburón hembra)

(9) El toro (cf. La vaca)

(10)El gusano (cf. El gusano hembra)

(11) El mosquito (cf. El mosquito hembra)

(12) El profeta (cf. La profetisa) 
Cuarenta y tres (43) informantes han fallado al dar la forma femenina de la palabra “carnero" en el caso de (6). Veintinueve (29) han escrito "La carnera”, 5 "La cabra”, 1 “La yerno”, 1 “La yerna”, 1 “La hembra” y 6 no escribieron nada.

Hemos encontrado 43 errores en el caso de (7). 21 participantes dejaron la pregunta en blanco, 15 han puesto “La testiga”, cuatro “El testigo” y tres “La hembra”. Estos errores resultan del desconocimiento de los sustantivos epicenos o de proceso de regularización. Cuarenta y tres (43) fallos han sido registrados en el caso de (8). 15 informantes dejaron la pregunta en blanco, 13 escribieron “La tiburón”, 14 “La tiburona” y 1 “La turba”.

Cuarenta y dos (42) participantes no acertaron en dar la hembra del toro (9). 20 dejaron la pregunta en blanco; 10 pusieron “La toro”, 7 “La tora”, 4 “La hembra” y 1 “La corrida”. Se han documentado 42 errores en el caso de (10). 22 informantes han puesto "La gusana”, 4 “La gusano” y 16 no rellenaron nada.

Son 42 los errores encontrados en el caso de (11). 19 participantes no escribieron nada; 14 escribieron "La mosquita”, 8 “La mosquito” y 1 “La mosca”.

Hemos registrado 42 fallos en la forma femenina de profeta (12). 30 han puesto "La profeta”, 3 “La profetesa”, 1 “La profete” y 1 “La profetera”. 7 dejaron la pregunta en blanco.

\section{Prueba 3: Pon en plural}

El número de errores documentados en la prueba 3 es de 667 errores que se distribuyen de la siguiente manera: los becarios: 137 errores; los no becarios: 530 errores.

(13) El pánico (cf. El pánico; francés la panique) 
(14)El tictac (cf. Los tictacs; francés les tictac)

(15)El gay (cf. Los gais)

(16) El no (cf. Los noes; francés les non)

(17) La a (cf. Las aes; francés les $a$ )

Son 43 los errores recogidos en el caso de (13). Veintiocho (28) informantes pusieron “Los pánicos”, 7 “Los panicos” y 8 no pusieron nada. Estos fallos pueden deberse a la generalización de la formación del plural o a la ignorancia.

Hemos encontrado 42 errores en el caso de (14). Treinta y dos (32) informantes pusieron “Los tictac”, 7 “Los tictaces” y 3 “Los tictaques”. Son incorrecciones debidas a la influencia del francés, a la generalización de la formación del plural, pero también a la arbitrariedad de la gramática.

Cuarenta y dos (42) informantes no han tenido aciertos en el caso de (15). Catorce (14) no contestaron a la pregunta; 8 han puesto “Los gays”, 6 “Los gay”, 6 “Los gayes”, 3 “Los gaies”, 2 “Los gaes”, 1 “Los gai”, 1 “Los guayes” y 1 “Los guapas”.

Se han registrado en el caso de (16) 42 errores, entre los cuales 39 errores se refieren a “Los no” y tres errores a "Los nos”.

Hemos registrado 42 errores en el caso de (17). 39 informantes pusieron "Las a” y tres “Las as”.

Los fallos en los casos de (16) y (17) se achacan a la imposición del plural en francés (nombre masculino invariable) y a la generalización de la formación del plural en español. 


\section{Prueba 4: Rodea la forma correcta}

El estudio del corpus nos ofrece 322 casos de incorrecciones, producidos por los becarios (45 errores) y los no becarios (277 errores).

(18) $(\mathrm{El} / \mathrm{La})$ rosa es mi color preferido. (cf. El; francés le rose)

(19) ¿Tienes (un/una) cortaúñas? (cf. Un; francés un coupe-ongles)

(20) ¿Es (un/una) gorila macho o hembra? (cf. un)

(21) Pon el plato en (el/la) microondas. (cf. el)

(22)(El/La) avestruz es un ave muy fuerte. (cf. El; francés l'autruche (sustantivo femenino))

Treinta y nueve (39) participantes prefirieron el artículo femenino al masculino en el caso de (18).

Se han producido 33 errores en el caso de (19); 31 errores en el caso de (20) y 31 fallos en el caso de (21). Los fallos en (19), (20) y (21) pueden atribuirse a la generalización de la formación del femenino en sustantivos.

Hemos localizado 29 errores en el caso de (22) que resultan de la imposición del género de la palabra francesa por asociación.

\section{Prueba 5: Sustituye las palabras subrayadas por una sola palabra.}

Los 79 errores registrados en la prueba 5 se reparten entre los becarios (8 errores) y los no becarios (71 errores).

(23)En mi clase hay un francés y dos francesas. (cf. tres franceses) 
(24)El rey y la reina de España viven en Madrid. (cf. reyes)

(25)El príncipe y la princesa de Asturias presiden un premio internacional. (cf. los príncipes)

Veintiún (21) informantes han desacertado en el caso de (23). Nueve han escrito “francés”, 3 “francófonos”, 2 “extranjeros”, 2 “nationalidad”, 2 “ellos”, 1 “francesas” y 2 no contestaron a la pregunta.

Se han encontrado 19 errores en el caso de (24). 2 participantes han puesto "reinos", 2 “reines”, 2 “reys”, 2 “la familia royal”, 2 “ellos”, 1 “reynado”, 1 “dinastía”, 1 “la reina”, 1 “el palacio real” y 5 no contestaron a la pregunta.

Son 15 los errores localizados en el caso de (25). 4 han escrito "hijos del rey y reina”, 2 “ellos”, 1 “princios”, 1 “prince, princese”, 1 “reyes”, 1 “princesa”, 1 “principios” y 4 no contestaron a la pregunta.

En resumidas cuentas, podemos decir que, según los resultados conseguidos en los análisis de errores realizados, tanto para los becarios como los no becarios, los errores más numerosos y relevantes aparecen en la segunda prueba, seguida por la tercera y la primera. La quinta prueba es la que presenta menos problemas. Los becarios han tenido mejores rendimientos que los no becarios en todas las pruebas. La tabla que sigue da muestra de ello, recogiendo la frecuencia de los aciertos y los desaciertos por prueba y por grupo. 
Tabla 3. Frecuencia de los aciertos y los desaciertos

\begin{tabular}{lccccc}
\hline \multicolumn{5}{c}{ BECARIOS } \\
\hline \multicolumn{7}{c}{$\mathrm{N}^{\mathrm{o}}$ de aciertos } & \multicolumn{1}{c}{$\mathrm{N}^{\mathrm{o}}$ de desaciertos } & $\mathrm{N}^{\mathrm{o}}$ total de ítems \\
\hline Prueba 1 & 268 & $72,43 \%$ & 102 & $27,57 \%$ & 370 \\
Prueba 2 & 47 & $24,74 \%$ & 143 & $75,26 \%$ & 190 \\
Prueba 3 & 63 & $31,50 \%$ & 137 & $68,50 \%$ & 200 \\
Prueba 4 & 165 & $78,57 \%$ & 45 & $21,43 \%$ & 210 \\
Prueba 5 & 52 & $86,67 \%$ & 8 & $13,33 \%$ & 60 \\
Total & 595 & $57,77 \%$ & 435 & $42,23 \%$ & 1030 \\
\hline \multicolumn{5}{c}{ NO BECARIOS } \\
\hline Prueba 1 & 795 & $61,39 \%$ & 500 & $38,61 \%$ & 1295 \\
Prueba 2 & 136 & $20,45 \%$ & 529 & $79,55 \%$ & 665 \\
Prueba 3 & 170 & $24,29 \%$ & 530 & $75,71 \%$ & 700 \\
Prueba 4 & 458 & $62,31 \%$ & 277 & $37,69 \%$ & 735 \\
Prueba 5 & 139 & $66,19 \%$ & 71 & $33,81 \%$ & 210 \\
Total & 1698 & $47,10 \%$ & 1907 & $52,90 \%$ & 3605 \\
\hline
\end{tabular}

En general, los participantes hombres han rendido más y mejor que las mujeres en ambos grupos, es decir, en el grupo de los becarios, los hombres han trabajado mejor que las becarias y sucede lo mismo en el grupo de los no becarios, si bien, la diferencia de media de error entre los becarios $(40,75)$ y las becarias $(45,34)$ es más evidente que la del grupo de los no becarios (los hombres $=53,92$ y las mujeres $=54,81$ ). Los estudiantes con mejor rendimiento, según el análisis de los datos, son los becarios hombres, seguidos por las becarias y los no becarios hombres. Las no becarias son las que tienen peores resultados, especialmente las dos participantes casadas. La tabla 4 ilustra la distribución y comparación del promedio de errores por género. 
Tabla 4. Distribución y comparación de la media de errores por género

\begin{tabular}{lcccc}
\hline \multicolumn{5}{c}{ Promedio de errores } \\
\hline Becarias & Becarios & No becarias & No becarios \\
\hline Prueba 1 & 11,67 & 8 & 14,86 & 13,31 \\
\hline Prueba 2 & 14,17 & 14,50 & 14,95 & 15,38 \\
\hline Prueba 4 & 14,17 & 13 & 14,86 & 15,62 \\
\hline Prueba 5 & 5 & 3,75 & 8,05 & 7,69 \\
\hline Total & 0,33 & 1,50 & 2,09 & 1,92 \\
\hline
\end{tabular}

\section{Discusión de los hallazgos}

La concordancia de género y número en español se percibe como fácil, asequible para la mayoría de los participantes (68,89\%). Parece posible que estos resultados se deban a la afinidad, la poca distancia lingüística entre el castellano y el francés, así como al estatus de este último en el país. Además, el hecho de que, según la cultura lingüística del país, se privilegia mucho más la eficacia comunicativa, la comprensibilidad del mensaje; se piensa que un error en la flexión nominal no impide la comprensión de la oración.

Pese al tiempo dedicado al castellano los participantes siguen atascándose con las normas gramaticales más básicas de la flexión nominal; sobre todo no son conscientes de las excepciones. Este resultado indica que, a pesar de ser estudiantes avanzados en una lengua siempre hay algunos "bolsillos" gramaticales que les hacen fracasar. Corrobora las investigaciones de algunos expertos (Bartning, 2000; Notario, 2001; Lambelet, 2012; Martín Jiménez, 2014) que señalan que los conocimientos gramaticales de los aprendientes no están afianzados, por quedarse en un nivel implícito. Asimismo, sugiere que los participantes solo aprenden las reglas gramaticales de manera más o menos memorística; solamente aprenden con la mera meta de aprobar exámenes, obtener títulos. 
Aun así, no se debe embrollar ambos actos, ya que induce a exclusión y restringe enormemente el modo de abordar el complejo proceso de enseñanza-aprendizaje de las lenguas extranjeras. Efectivamente, evaluar, examinar y calificar son actos distintos.

La prueba 2 ha sido la más difícil confirmando las hipótesis de Bartning (2000) y Lambelet (2012) que indican que tanto aprendientes de nivel intermedio como avanzado siguen teniendo problemas con la determinación de género en francés. Estos resultados confirman lo ya encontrado por Eddington y Hualde (2008); Ferreira Cabrera y Elejalde Gómez (2017) y Zuheros Garrido y Marrero Aguiar (2019).

Los fallos registrados en la prueba 1 patentizan el deseo de estos participantes de expresar la regularidad en la concordancia de género. De hecho, quieren reducir la gramática a algo más sencillo mediante la estrategia de simplificación de la regla morfológica (cf. $\underline{e l}$ nao; $\underline{e l}$ postal; $\underline{e l}$ miel, etc.); quieren fomentar la sencillez e impulsar la eficiencia. Este hallazgo sugiere que los informantes son sensibles a las terminaciones de los sustantivos al igual que los hispanohablantes de Caffarra y Barber (2015) y los hablantes del español como L2 de Halberstadt, Valdés Kroff y Dussias (2018). Resultados similares han sido obtenidos por Dewaele y Véronique (2001), Ayoun (2010) que consideran el género como uno de los elementos más delicados de la adquisición del francés como LE, incluso del francés como L1.

Los errores que aparecen en el corpus pueden producirse por la interferencia de la lengua francesa, estrategia muy utilizada, consciente o inconscientemente, por los discentes francófonos, por la interferencia de la lengua meta, debido al uso regido por reglas generales, al uso específico de la flexión nominal, la arbitrariedad de la lengua o por una interacción de estas dos interferencias (Sossouvi, 2016b, 2017a). Muchos estudiosos sostienen que cuanto menos es la distancia lingüística entre las lenguas de contacto 
implicadas, más facilidades se encuentran, especialmente en las primeras etapas de adquisición. Este planteamiento queda reflejado en el análisis de errores de nuestro corpus de datos, ya que la poca distancia entre la lengua francesa y la española, por pertenecer a la misma familia lingüística, y el hecho de que en algunas lenguas nacionales o autóctonas no haya flexión nominal como en las lenguas romances, aunque existe mecanismos para expresar este aspecto gramatical, hacen que los aprendientes presenten cierta facilidad, pero a veces resistencia, en el uso de la flexión nominal. En consecuencia, es más trascendental la afinidad entre las lenguas de contacto implicadas, es decir, tanto la cercanía estructural como la sensación psicológica entre las lenguas (Cenoz, 2003; Foote, 2009; Montrul, Dias y Santos, 2011; Sossouvi, 2014, 2017a; Rothman, 2015; Palacios, 2017; Lambert, 2019), y el estatus de la lengua en el país (Sossouvi, 2016a, 2017b) que el orden de adquisición de las diversas lenguas.

Este estudio apoya la investigación de Sossouvi (2017a) que llegó a la conclusión de que el francés podría ser la lengua de referencia, la lengua puente para el aprendizaje significativo de ELE en el país, incluso para promover nuevos aprendizajes. Igualmente, este trabajo muestra la fuerza de la lengua de instrucción y de la afinidad entre las lenguas en contacto. Por otro lado, sugiere que, aunque hay similitudes importantes entre lenguas, también hay factores que las diferencian y conducen inexorablemente a la perplejidad.

La influencia de las lenguas locales como el fongbe, el gungbe, etc. ha sido insignificante, especialmente en estas categorías gramaticales, debido a la situación precaria de estas lenguas en el sistema educativo, además de su pertenencia a otra familia lingüística, no indoeuropea.

En suma, los participantes de este trabajo cometieron errores interlingüísticos por la interferencia de las construcciones sintácticas de la lengua francesa (u otras lenguas); 
errores intralingüísticos (errores de desarrollo), esto es, por el mismo proceso de aprendizaje de la lengua castellana, como por ejemplo las peculiaridades del español, la aplicación incorrecta de reglas gramaticales o la generalización a todos los contextos y por fin, otros tipos de errores, tales como errores inducidos. De igual forma, estos fallos pueden ocurrir por dificultades procedimentales que tienen que ver con un conocimiento incompleto de la flexión nominal tanto en español como en francés y déficit relacionado con la disponibilidad de recursos de la memoria. De hecho, la interdependencia de las competencias lingüísticas (cf. Dressler y Kamil, 2006; Ganuza y Hedman, 2019) parece muy importante en la adquisición y uso de una lengua adicional.

Si se toman en conjunto los resultados de todas las pruebas, los estudiantes becados son mejores que los no becarios. Este hallazgo podría deberse a las condiciones de estudio, de vida y de becario o no. Los diez becarios están entre los mejores del aula, por estar entre los diez primeros de los que superaron la prueba nacional de acceso a la escuela universitaria, y hace falta cumplir unos requisitos académicos ${ }^{1}$ para seguir disfrutando de la beca el curso siguiente.

Por sexos, los hombres tienen una mejor tasa de éxito. Puede deberse a la estructura social, los procesos de socialización, la desigualdad de género en la sociedad beninesa, ya que las mujeres enfrentan grandes desventajas en ella. Esto se puede observar en prácticas discriminatorias, normas sociales y patrones de conducta o comportamientos que las conducen a menos progresos en su trayectoria escolar o académica. A pesar de ser estudiantes, las muchachas de esa edad dedican varias horas al día a los quehaceres

\footnotetext{
1 A modo de ejemplo, deben aprobar en su totalidad todas las asignaturas o créditos, necesitan una nota media superior o igual a 12/20 y no pueden repetir curso so pena de denegación de beca.
} 
domésticos y menos horas a las tareas escolares en casa que los muchachos. Generalmente, tienen menos confianza en sí mismas para enfrentar las pruebas académicas, y tienden a tener un sentimiento de ansiedad hacia las pruebas de este tipo (de ahí la necesidad de un coaching pedagógico en las escuelas) y hacia su futuro hogar como esposas y madres.

De hecho, la sociedad beninesa misma ya ha puesto los atributos, y un ejemplo de ello, como la denominación de la mujer y del hombre en fongbe: la primera es nyว̆nù xwésì, es decir un agua potable, algo precioso que se debe beber con moderación, la cuidadora del hogar, de niños, de la familia en general; mientras que el hombre se llama súnnù glegbenù, es decir, el defensor y protector de la casa, de la familia ante las amenazas; el proveedor de pan, de comida, el que da el sustento para la casa. Por consiguiente, por medio de los procesos de socialización, cada miembro de la sociedad beninesa va adaptando una identidad de género, siguiendo las disposiciones culturales y prácticas relacionadas a su sexo. Es decir, a esa edad, la mayoría se preocupa más por casarse, la maternidad y formar su propia familia. Estos datos parecen apoyar la investigación previa de Lin y Sossouvi (2017) que apuntó que los hombres resultaron mejores que las mujeres en la alternancia de los tiempos del pasado en castellano.

Los resultados de las dos participantes madres confirman los ya encontrados por Ion, Durán-Bellonch y Bernabeu Tamayo (2013), que indican que las responsabilidades familiares pueden frenar la carrera académica de las mujeres. De este modo, estudios, casamiento y maternidad son una combinación difícil de manejar.

No obstante, el hecho de que las becarias hayan superado a los no becarios indica que, las mujeres puestas en las mismas condiciones, con las mismas oportunidades, son tan capaces como los hombres. El género femenino es capaz de realizar las mismas labores 
que el masculino, sean estas de la sustancia que fueran. Las mujeres, resumidamente, son igual de valiosas, brillantes, talentosas y capaces que los hombres.

\section{Implementaciones didácticas}

Los resultados de este estudio coinciden con los de Bybee (2008) y MacWhinney (2008) e indican que resulta difícil para los aprendientes de ELE en un contexto formal obtener la característica de un contexto de español como lengua materna, e incluso como lengua de herencia. Las implicaciones derivadas del análisis cualitativo y cuantitativo de los fallos obligan a utilizar una metodología adecuada de enseñanza-aprendizaje de la flexión nominal, teniendo en cuenta los errores analizados, las estrategias utilizadas, las interferencias y las insuficiencias didácticas. Las dificultades observadas en la adquisición y el uso de la flexión nominal, así como la complejidad de su descripción imponen la necesidad de crear materiales específicos centrados en una metodología de enseñanza en consonancia con las necesidades y las características de los aprendientes benineses, con el enfoque intercultural. De igual modo, no solo se debe tener en cuenta el contexto de aprendizaje, sino también los efectos del input de castellano. De acuerdo con estudiosos como Sossouvi (2016a: 57), el MCER contextualizado podría ofrecer un gran potencial educativo para ellos. Así, el discente tendrá más exposición a una mayor cantidad de input de castellano, más implicación en actividades de comprensión, interacción y producción.

De esta forma, el aprendizaje de la flexión nominal no solo consistiría en repetir ejercicios para poder llegar a una sistematización de su uso; la flexión nominal debe ser enseñada, además, de forma contextualizada con dispositivos tecnológicos, junto con las explicaciones gramaticales correspondientes, basadas en un enfoque no meramente 
descriptivo, sino también integral, para poder conocer sus diversos usos y valores. Teniendo en cuenta la complejidad de la descripción del género y número de los sustantivos, otra posible propuesta para remediar sería la memorización del género y número de los sustantivos a medida que el aprendiente aprende el léxico. Por lo menos, memorizar cuando el género y el número resultan distintos de los de las L1s, sobre todo, las numerosas excepciones y completar con actividades de conceptualización. Desde otro ángulo, es conveniente fomentar la consciencia lingüística o la consciencia sobre las diferentes lenguas de su repertorio lingüístico, con el fin de poder controlar las interferencias a nivel de la flexión nominal y otros. Igualmente, España puede establecer y consolidar acuerdos de cooperación con el gobierno beninés en la formación de los (futuros o) profesionales de la lengua española, tanto a distancia como presencialmente (Sossouvi, 2017b).

Por otra parte, el estudio del castellano como LE a edades tempranas podría generar innumerables ventajas y especialmente la mejora de la competencia comunicativa. El tiempo de instrucción puede afectar positivamente el conocimiento de este aspecto gramatical. Asimismo, se posibilita una educación globalizada, intercultural, fomentando el conocimiento en aspectos socioculturales del español. Así, los estudiantes podrán incorporar las reglas gramaticales a su uso diario de manera natural.

Por fin, la incorporación de la perspectiva de género en el sistema educativo del país, en especial, en la formación del profesorado será de mucha utilidad para la mejora del pleno desarrollo del país. 


\section{Reflexiones finales}

De lo expuesto hasta aquí, podemos decir que los participantes siguen teniendo problemas con la concordancia de género y número, sobre todo las excepciones que parecen ser la categoría más difícil. Fallaron más en las pruebas 2, 3 y 1. Estos errores se deben a varios factores, entre los cuales su competencia plurilingüe, los calcos de la estructura del francés, las dificultades intrínsecas a la propia lengua española, la arbitrariedad de algunas reglas gramaticales, el contexto de enseñanza-aprendizaje de la lengua meta, un aprendizaje deficiente del castellano, una competencia defectuosa en la propia lengua francesa, la falta de inversión del estudiantado, los materiales didácticos, los tipos de tareas utilizadas, distracciones, entre otros.

Tanto los estudiantes varones como las femeninas pueden rozar la excelencia durante sus estudios universitarios, lo esencial es asegurarles buenas condiciones para los estudios, la igualdad de condiciones, de deseos y de opiniones. En síntesis, queda confirmada nuestra hipótesis y precisa una didáctica contextualizada crítica del español, una colaboración de España e incluso de los países hispanohablantes en la formación de profesores de español en las escuelas secundarias, una creación de mecanismos contra la discriminación de género, la concesión de más becas y ayudas universitarias, principalmente a las mujeres, la reafirmación o replanteamiento del concepto de mujer, para darles más impulso académico, profesional, y para que lleguen así mejor preparadas para asumir su carrera profesional en el futuro.

En definitiva, esta investigación arroja alguna luz sobre la complejidad de la adquisición de la flexión nominal por discentes francófonos de África, presenta hasta cierto punto, herramientas teóricas y metodológicas que posibiliten arriesgarse en ese escollo, así como 
exhorta a diversificar las metas de la evaluación, impulsa a una evaluación formativa e insiste en la calidad del aprendizaje efectivo de los aprendientes de lenguas extranjeras.

\section{Referencias bibliográficas}

Aaron, Jessi Elana (2015). Lone English-origin nouns in Spanish: The precedence of community norms. International Journal of Bilingualism, 19(4), 459-480. doi: $10.1177 / 1367006913516021$

Aragonés, Luis \& Palencia, Ramón (2009). Gramática de uso del español. Teoría y práctica B1-B2. Madrid: Ediciones SM.

Ayoun, Dalila (2010). Corpus data: Shedding the light on French grammatical gender...or not. EUROSLA Yearbook, 10, 119-141.

Bartning, Inge (2000). Gender agreement in L2 French: Pre-advanced vs advanced learners. Studia Linguistica, 54(2), 225-237.

Beatty-Martínez, Anne L. \& Dussias, Paola E. (2019). Revisiting masculine and feminine grammatical gender in Spanish: Linguistic, psycholinguistic, and neurolinguistic evidence. Frontiers in Psychology, 10, 1-11. doi: 10.3389/fpsyg.2019.00751

Bellamy, Kate, Parafita Couto, M. Carmen \& Stadthagen-Gonzalez, Hans (2018). Investigating gender assignment strategies in mixed Purepecha-Spanish nominal constructions. Languages, 3(28), 1-17. doi: 10.3390/languages3030028

Bosque, Ignacio \& Demonte, Violeta (Dirs.) (1999). Gramática descriptiva de la lengua española. Vol. 1 y Vol. 3. Madrid: Espasa - Real Academia Española, colección Nebrija y Bello. 
Bybee, Joan (2008). Usage-based grammar and second language acquisition. En Peter Robinson \& Nick C. Ellis (Eds.), Handbook of Cognitive Linguistics and Second Language Acquisition (pp. 216-236). New York, London: Routledge.

Caffarra, Sendy \& Barber, Horacio A. (2015). Does the ending matter? The role of gender-to-ending consistency in sentence reading. Brain Research, 1605, 83-92. doi: 10.1016/j.brainres.2015.02.018

Cenoz, Jasone (2003). The role of typology in the organization of the multilingual lexicon. En Jasone Cenoz, Britta Hufeisen \& Ulrike Jessner (Eds.), The Multilingual Lexicon (pp. 103-116). Dordrecht: Kluwer.

Clegg, Jens H. \& Waltermire, Mark (2009). Gender assignment to English-origin nouns in the Spanish of the Southwestern United States. Southwest Journal of Linguistics, 28(1), 1-17.

Corder, S. Pit (1981). Error analysis and interlanguage. Oxford: Oxford University Press.

Dewaele, Jean-Marc \& Véronique, Daniel (2001). Gender assignment and gender agreement in advanced French interlanguage: A cross-sectional study. Bilingualism: Language and Cognition, 4(3), 275-297. doi: 10.1017/S136672890100044X

Djandué, Bi Drombé (2012). La influencia de los estereotipos en el aprendizaje del Español como Lengua Extranjera (E/LE) en Costa de Marfil. redELE, 24.

Dressler, Cheryl \& Kamil, Michael L. (2006). First-and second-language literacy. En Diane August \& Timothy Shanahan (Eds.), Developing Reading and Writing in Second-Language Learners: Lessons from the Report of the National Literacy 
Panel on Language-Minority Children and Youth (pp.197-238). Mahwah, NJ: L. Erlbaum Associates.

Eddington, David \& Hualde, José Ignacio (2008). El abundante agua fría: hermaphroditic Spanish nouns. Studies in Hispanic and Lusophone Linguistics, 1(1), 5-32. doi: 10.1515/shll-2008-1004

Fadaïro, Dominique (2001). Parlons fon. Langue et culture du Bénin. París: L’Harmattan. Ferreira Cabrera, Anita \& Elejalde Gómez, Jéssica (2017). Análisis de errores recurrentes en el Corpus de Aprendices de Español como Lengua Extranjera, CAELE. RBLA, Belo Horizonte, 17(3), 509-537.

Foote, Rebecca (2009). Transfer in L3 acquisition: The role of typology. En Yan-kit Ingrid Leung (Ed.), Third Language Acquisition and Universal Grammar (pp. 89114). Bristol, Buffalo, Toronto: Multilingual Matters.

Franceschina, Florencia (2001). Morphological or syntactic deficits in near-native speakers? An assessment of some current proposals. Second Language Research, 17(3), 213-247.

Franco, María Olga (1999). Adquisición del género en lengua francesa por niños españoles. Infancia y Aprendizaje, 88, 57-74. doi: 10.1174/021037099760246617

Ganuza, Natalia \& Hedman, Christina (2019). The impact of mother tongue instruction on the development of biliteracy: Evidence from Somali-Swedish bilinguals. Applied Linguistics, 40(1), 108-131.

Gómez Torrego, Leonardo (2011). Gramática didáctica del español. Madrid: Ediciones SM. 
Gonzalez, Paz, Mayans, Damaris \& van den Bergh, Huub (2019). Nominal agreement in the interlanguage of Dutch L2 learners of Spanish. IRAL - International Review of Applied Linguistics in Language Teaching, 1-20. doi: 10.1515/iral-2017-0174

Greenberg, Joseph H. (1963). The Languages of Africa. International Journal of American Linguistics, 29(1), part 2.

Halberstadt, Lauren, Valdés Kroff, Jorge R. \& Dussias, Paola E. (2018). Grammatical gender processing in L2 speakers of Spanish: The role of cognate status and gender transparency. Journal of Second Language Studies, 1(1), 5-30. doi: 10.1075/jsls.17023.hal

Ion, Georgeta, Durán-Bellonch, Maria del Mar \& Bernabeu Tamayo, Maria Dolors (2013). El profesorado y su percepción sobre la igualdad de género en la Universidad. Teaching staff and their perception about gender equality at university. Revista Complutense de Educación, 24(1), 123-140.

Jake, Janice L., Myers-Scotton, Carol \& Gross, Steven (2002). Making a minimalist approach to codeswitching work: Adding the matrix language. Bilingualism: Language and Cognition, 5(1), 69-91. doi: 10.1017/S1366728902000147

Lambelet, Amelia (2012). L'apprentissage du genre grammatical en langue étrangère : à la croisée des approches linguistiques et cognitives. Tesis doctoral. Fribourg: Université de Fribourg.

Lambert, Craig (2019). Referent Similarity and Nominal Syntax in Task-Based Language Teaching. Gateway East: Springer. 
Lew-Williams, Casey \& Fernald, Anne (2010). Real-time processing of gender-marked articles by native and non-native Spanish speakers. J Mem Lang, 63(4), 447-464. doi: 10.1016/j.jml.2010.07.003.

Lin, Mei-Chih \& Sossouvi, Laurent-Fidèle (2017). Escollos en la alternancia de los tiempos del pasado en español por estudiantes benineses. Revista Internacional de Investigación y Docencia (RIID), 2(3), 7-15. Recuperado de http://onlinejournal.org.uk/index.php/riid/issue/view/19/showToc. DOI: http://dx.doi.org/10.19239/riidv2n3p7

MacWhinney, Brian (2008). A unified model. En Peter Robinson \& Nick C. Ellis (Eds.), Handbook of Cognitive Linguistics and Second Language Acquisition (pp. 341371). New York, London: Routledge.

Martín Jiménez, Ma Amparo (2014). Planteando problemas de gramática a los alumnos de Filología. Presenting grammar problems to students of Philology. Tejuelo, Monográfico, 10, 143-162.

Montrul, Silvina, de la Fuente, Israel, Davidson, Justin \& Foote, Rebecca (2013). The role of experience in the acquisition and production of diminutives and gender in Spanish: Evidence from L2 learners and heritage speakers. Second Language Research, 29(1), 87-118.

Montrul, Silvina, Dias, Rejanes \& Santos, Hélade (2011). Clitics and object expression in the L3 acquisition of Brazilian Portuguese. Structural similarity matters for transfer. Second Language Research, 27(1), 21-58. 
Notario, Gema (2001). Conceptos gramaticales de los alumnos de secundaria: el sujeto. En Anna Camps (Coord.), El aula como espacio de investigación y reflexión: investigaciones en didáctica de la lengua (pp. 181-194). Barcelona: Graó.

Palacios, Azucena (Coord.) (2017). Variación y cambio lingüístico en situaciones de contacto. Madrid: Iberoamericana Vervuert.

Parafita Couto, M. Carmen, Munarriz, Amaia, Epelde, Irantzu, Deuchar, Margaret \& Oyharçabal, Beñat (2015). Gender conflict resolution in Spanish-Basque mixed DPs. Bilingualism: Language and Cognition, 18(2), 304-323. doi: 10.1017/S136672891400011X

RAE \& ASALE (2010). Nueva gramática de la lengua española MANUAL. Madrid: Espasa.

RAE (2018). Libro de estilo de la lengua española según la norma panhispánica. Madrid: Espasa.

Rothman, Jason (2015). Linguistic and cognitive motivations for the Typological Primacy Model (TPM) of third language (L3) transfer: Timing of acquisition and proficiency considered. Bilingualism: Language and Cognition, 18(2), 179-190. doi:10.1017/S136672891300059X

Rothman, Jason, González Alonso, Jorge \& Miller, David (2018). The acquisition of second language Spanish morphosyntax. En Kimberly L. Geeslin (Ed.), The Cambridge Handbook of Spanish Linguistics (pp. 689-715). Cambridge: Cambridge University Press. doi: 10.1017/9781316779194.032

Smead, Robert N. (2000). On the assignment of gender to Chicano anglicisms: Processes and results. Bilingual Review/ Revista Bilignue, 25(3), 277-297. 
Société des Missions Africaines (2012). Eléments de Grammaire de la langue Fon (Sud Bénin). Cotonou: Société des Missions Africaines.

Sossouvi, Laurent-Fidèle (2004). Análisis de errores de los alumnos benineses de español como lengua extranjera. Tesis doctoral. Alcalá de Henares: Universidad de Alcalá.

Sossouvi, Laurent-Fidèle (2014). La lengua castellana en Benín: tendencias actuales después de seis décadas. En Javier Serrano Avilés (Ed.), La situación del español en África subsahariana (pp. 100-126). Madrid: Catarata.

Sossouvi, Laurent-Fidèle (2016a). Enseñanza y aprendizaje del ELE en Benín: ¿qué puede aportar la adaptación del MCER? / Teaching and learning of SFL in Benin: What can provide the adaptation of the CEFR?. Tejuelo, 23, 36-62. Recuperado de http://mascvuex.unex.es/revistas/index.php/tejuelo/index. doi: 10.17398/19888430.23.1.36

Sossouvi, Laurent-Fidèle (2016b). Hacia una caracterización de la producción oral espontánea de los estudiantes universitarios benineses de ELE. European Scientific Journal, $\quad 35(12), \quad$ 209-228. Recuperado de eujournal.org/index.php/esj/article/download/8514/8183

Sossouvi, Laurent-Fidèle (2017a). Influencia translingüística: en busca de una lengua de referencia en la interlengua oral de estudiantes benineses de ELE. e-AESLA Revista digital, 31-87. $\quad$ 71 $\quad$ Recuperado de http://cvc.cervantes.es/lengua/eaesla/pdf/03/06.pdf

Sossouvi, Laurent-Fidèle (2017b). Replantear la enseñanza-aprendizaje de ELE en Benín: los posibles aportes de la adaptación del MCER. Balti: Editorial Académica Española. 
Torijano, J. Agustín (2008). El estudio de los determinantes en aprendices lusohablantes de español. DICENDA. Cuadernos de Filología Hispánica, 26, 235-257.

Yao, Firmin (2015). Préstamos del español al nouchi hablado en Costa de Marfil. Revue Baobab, 17, 61-76.

Zuheros Garrido, Laura \& Marrero Aguiar, Victoria (2019). La edad en la adquisición de la morfología nominal de género y número del español por parte de sinohablantes. ELUA, 33, 233-248. doi: 10.14198/ELUA2019.33.12 